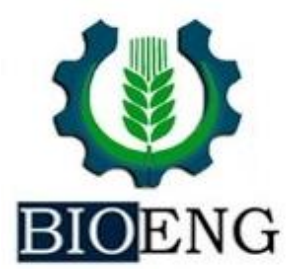

\title{
ESTIMATIVA DA EVAPOTRANSPIRAÇÃO DE REFERÊNCIA DIÁRIA EM FORMIGA, MG, BRASIL
}

\author{
L. R. da Paz; M. S. Thebaldi*
}

UNIFOR - Centro Universitário de Formiga, Formiga, MG, Brasil

Article history: Received 07 February 2018; Received in revised form 17 February 2018; Accepted 19 February 2018; Available online 27 March 2018.

\section{RESUMO}

Quantificar a evapotranspiração é de grande importância no gerenciamento dos recursos hídricos, modelagem hidrológica e meteorológica e também no manejo hídrico sustentável de culturas irrigadas. Por isso, este trabalho teve por objetivo estimar a evapotranspiração de referência $\left(\mathrm{ET}_{0}\right)$ diária, para o município de Formiga - MG, utilizando o método padrão de Penman-Monteith FAO e outros três métodos empíricos. A estimativa da evapotranspiração de referência $\left(\mathrm{ET}_{0}\right)$ diária foi realizada a partir de dados disponibilizados pelo INMET, coletados por estação meteorológica automática, entre os anos de 2007 e 2014. Além disso, foram ajustadas as constantes empíricas dos métodos de Hargreaves, Makkink e Camargo, de forma que estas se adequassem às condições locais. Para a análise do desempenho dos métodos, foram considerados os índices de confiança (c), de concordância (d), coeficientes de correlação (r) e determinação $\left(\mathrm{R}^{2}\right)$; erro absoluto médio (EAM) e raiz quadrada do erro quadrado médio (RQEM). Verificou-se que o método de Makkink, após ajuste, foi o que obteve melhores estimativas para as condições de Formiga, MG.

Palavras-chave: avaliação de modelos, elementos meteorológicos, gerenciamento de recursos hídricos.

\section{ESTIMATE OF DAILY REFERENCE EVAPOTRANSPIRATION AT FORMIGA, MG, BRAZIL}

\begin{abstract}
The evapotranspiration quantification has a great importance on water resources management, hydrological and meteorological modeling and on sustainable water management of irrigated crops. Therefore, this paper aims to estimate the daily reference evapotranspiration $\left(\mathrm{ET}_{0}\right)$, in Formiga, MG, Brazil, using the Penman-Monteith's Method under FAO standards and other three empirical methods. The daily reference evapotranspiration $\left(\mathrm{ET}_{0}\right)$ estimation was carried out from data provided by INMET, collected by an automatic weather station, between the years 2007 and 2014. In addition, the empirical constants of Hargreaves, Makkink and Camargo Methods were fit, to represent better the local conditions. For method's performance analysis, were considered: the reliability index (c), concordance index (d), correlation (r) and determination coefficients $\left(\mathrm{R}^{2}\right)$; mean absolute error (MAE) and square root of the mean square
\end{abstract}

msthebaldi@uniformg.edu.br 
error (SRME). It was found that the method of Makkink, after adjustment, produced the best estimates for daily reference evapotranspiration of the Formiga, MG

Keywords: models evaluation, meteorological elements, water resources management.

\section{INTRODUÇÃO}

O estudo da evapotranspiração é de suma importância no planejamento e manejo da irrigação e no gerenciamento e uso dos recursos hídricos, pois como elemento do balanço hídrico, é possível quantificar a disponibilidade hídrica das regiões. As atividades agrícolas consomem grandes quantidades de água, assim, esforços quanto ao uso racional e eficiente deste recurso natural têm sido empregados (LACERDA e TURCO, 2015).

Com o objetivo de padronizar sua estimativa, definiu-se o conceito de evapotranspiração de referência $\left(\mathrm{ET}_{0}\right)$, como aquela que ocorre em uma cultura hipotética, a qual possui uma altura fixa de $0,12 \mathrm{~m}$, albedo igual a 0,23 e resistência da superfície ao transporte de vapor de água igual a $70 \mathrm{~s} \mathrm{~m}^{-1}$. Desta maneira, determinouse que o método de Penman-Monteith seria o modelo padrão para estimativa da evapotranspiração de referência, por apresentar melhor desempenho em climas variados (ALLEN et al., 1998).

Este conceito foi originalmente introduzido para investigar a demanda evaporativa da atmosfera, independentemente do tipo de cultura,

\section{MATERIAL E MÉTODOS}

Os dados meteorológicos foram obtidos digitalmente junto ao Instituto Nacional de Meteorologia (INMET) sendo provenientes da estação meteorológica automática de Formiga - MG. A estação está localizada nas coordenadas latitude $20^{\circ} 27^{\prime} \mathrm{S}$ e longitude $45^{\circ} 27^{\prime} \mathrm{W}$, datum WGS 84, a $878 \mathrm{~m}$ de altitude.

O clima no município de Formiga MG, pela classificação de Köppen-Geiger, é fenologia e tratos culturais (ALENCAR et al., 2011).

Entretanto, o método de PenmanMonteith depende de muitas variáveis meteorológicas, que nem sempre estão disponíveis em todos os locais. Por isso, métodos mais simples e que requerem uma quantidade menor de variáveis meteorológicas, vem sendo buscados por se mostrarem satisfatórios e apresentarem boa aplicabilidade. Contudo, antes de utilizá-los deve-se verificar seu desempenho para o local em questão, e se for necessário calibrar os modelos a fim de reduzir erros de estimativas (PEREIRA et al., 2009).

Assim, o objetivo deste trabalho foi estimar a evapotranspiração de referência (ET0) diária, para o município de Formiga MG, utilizando o método padrão de PenmanMonteith FAO e os métodos empíricos de Hargreaves, Makkink e Camargo, além de realizar o ajuste das constantes empíricas destes três últimos, para as condições de Formiga - MG, avaliando o desempenho dos métodos empíricos de ET0, ajustados ou não, em relação ao método padrão de Penman-Monteith FAO.

Cwb (ALVARES et al., 2013), sendo temperado chuvoso, subtropical com inverno seco e verão quente. Possui duas estações bem definidas, apresenta uma temperatura média anual entre $21^{\circ} \mathrm{C}$ a $23^{\circ} \mathrm{C}$ e o índice pluviométrico anual é de $1400 \mathrm{~mm}$.

A série histórica avaliada abrangeu os anos de 2007 a 2014, porém, os dias que apresentavam falhas em qualquer variável do modelo foram descartados da análise. Assim, 
esses dias não foram utilizados para o cálculo da evapotranspiração de referência, o que totalizou 828 dias efetivos de dados trabalhados.

Para estimativa da evapotranspiração de referência de Formiga - MG, foram

$$
\mathrm{ET}_{0}=\frac{0,408 \times \Delta \times\left(\mathrm{R}_{\mathrm{n}}-\mathrm{G}\right)+\gamma \times \frac{900}{\mathrm{~T}+273} \times \mathrm{u}_{2} \times\left(\mathrm{e}_{\mathrm{s}}-\mathrm{e}_{\mathrm{a}}\right)}{\Delta+\gamma \times\left(1+0,34 \times \mathrm{u}_{2}\right)}
$$

Em que:

$\mathrm{ET}_{0}=$ evapotranspiração de referência (mm.dia-1);

$\mathrm{Rn}=$ radiação líquida na superfície da planta (MJ.m-2.dia-1);

$\mathrm{G}=$ densidade de fluxo de calor no solo (MJ.m-2.dia-1);

$\mathrm{T}=$ temperatura média do ar a $2 \mathrm{~m}$ de altura $\left({ }^{\circ} \mathrm{C}\right)$;

$\mathrm{u}_{2}=$ velocidade do vento a $2 \mathrm{~m}$ de altura (m.s-1);

$\mathrm{e}_{\mathrm{s}}=$ pressão de saturação de vapor $(\mathrm{kPa})$; utilizados os métodos Penman - Monteith FAO (Equação 1), Hargreaves, Camargo e Makkink, sendo o primeiro, o método padrão para cálculo da $\mathrm{ET}_{0}$ (ALLEN et al., 1998).

$$
\mathrm{ET}_{0}=0,408 \times 0,0023 \times\left(\mathrm{T}_{\text {méd }}+17,8\right) \times \sqrt{\left(\mathrm{T}_{\text {máx }}-\mathrm{T}_{\text {mín }}\right)} \times \mathrm{R}_{\mathrm{a}}
$$

Em que:

$\mathrm{R}_{\mathrm{a}}=$ radiação no topo da atmosfera $\left(\mathrm{MJ} \mathrm{m}^{-2}\right.$ $\left.\operatorname{dia}^{-1}\right)$.

$$
\mathrm{ET}_{\mathrm{X}}=\mathrm{F} \times \mathrm{R}_{\mathrm{Ax}} \times \mathrm{T}_{\mathrm{X}} \times \mathrm{X}
$$

Em que:

$\mathrm{R}_{\mathrm{Ax}}=$ radiação solar extraterrestre média do período, expressa em evaporação equivalente $\left(\mathrm{mm} \mathrm{dia}^{-1}\right)$;

$\mathrm{T}_{\mathrm{x}}=$ temperatura média do período $\left({ }^{\circ} \mathrm{C}\right)$;

$\mathrm{F}=$ fator de ajuste $=0,01$;

$$
\mathrm{ET}_{0}=0,61 \times \mathrm{W} \times \mathrm{R}_{\mathrm{S}}-0,12
$$

Em que:

$\mathrm{R}_{\mathrm{S}}=$ total diário de radiação solar medida em equivalente de evaporação $\left(\mathrm{mm} \mathrm{dia}{ }^{-1}\right)$; $\mathrm{e}_{\mathrm{a}}=$ pressão atual de vapor $(\mathrm{kPa})$;

$\Delta=$ declividade da curva de pressão de vapor $\left(\mathrm{kPa} .{ }^{\circ} \mathrm{C}-1\right)$;

$\gamma=$ constante psicrométrica $\left(\mathrm{kPa} .{ }^{\circ} \mathrm{C}-1\right) ; \mathrm{e}$

$0,408=$ fator de conversão para o termo $(\mathrm{Rn}$

- G), de MJ.m-2.dia-1 para mm.dia-1.

As equações padronizadas utilizadas para obtenção dos variáveis apresentados na Equação 1 são descritas em ALLEN et al. (1998). Já para o método Hargreaves, a ET0 é descrita pela Equação 2:
O valor de 0,0023 é um coeficiente adimensional, empírico, proposto para $\mathrm{o}$ método. Já a $\mathrm{ET}_{0}$ pelo método de Camargo pode ser estimada por meio da Equação 3:

$\mathrm{X}=$ número de dias do período, sendo utilizado neste trabalho 1 .

Para o método de Makkink, a $\mathrm{ET}_{0}$ é estimada pela Equação 4:

$\mathrm{W}=$ fator de ponderação (adimensional). 
Esse fator de ponderação pode ser calculado pela Equação 5:

$$
\mathrm{W}=\left(0,392+310^{-5} \times \mathrm{Z}\right)+0,0172 \times \mathrm{T}_{\text {méd }}-0,0001 \times \mathrm{T}_{\text {méd }}{ }^{2}
$$

Em que:

$\mathrm{Z}=$ altitude do local (m).

Para avaliação de desempenho dos modelos de Hargreaves, Camargo e Makkink, em relação ao método padrão foram utilizadas as seguintes estatísticas e indicadores: erro absoluto médio (EAM) Equação 6; raiz quadrada do erro quadrado médio (REQM) - Equação 7; índice de concordância (d) - Equação 8; índice de confiança (C) - Equação 9; coeficientes de correlação (r); e determinação $\left(\mathrm{R}^{2}\right)$; além dos coeficientes linear e angular da reta de regressão $\left(a_{R} \quad\right.$ e $\quad b_{R}$, respectivamente $)$ (WILLMOTT, 1982; CAMARGO e SENTELHAS, 1997).

$$
\begin{aligned}
& \text { EAM }=\mathrm{N}_{\mathrm{d}}^{-1} \sum_{\mathrm{i}=1}^{\mathrm{N}_{\mathrm{d}}}\left|\mathrm{P}_{\mathrm{i}}-\mathrm{O}_{\mathrm{i}}\right| \\
& \mathrm{REQM}=\left[\mathrm{N}_{\mathrm{d}}^{-1} \sum_{\mathrm{i}=1}^{\mathrm{N}_{\mathrm{d}}}\left(\mathrm{P}_{\mathrm{i}}-\mathrm{O}_{\mathrm{i}}\right)^{2}\right]^{0,5} \\
& \mathrm{~d}=1-\left[\frac{\sum_{\mathrm{i}=1}^{\mathrm{N}_{\mathrm{d}}}\left(\mathrm{P}_{\mathrm{i}}-\mathrm{O}_{\mathrm{i}}\right)^{2}}{\sum_{\mathrm{i}=1}^{\mathrm{N}_{\mathrm{d}}}\left(\left|\mathrm{P}_{\mathrm{i}}-\overline{\mathrm{O}}\right|+\left|\mathrm{O}_{\mathrm{i}}-\overline{\mathrm{O}}\right|\right)^{2}}\right] \\
& \mathrm{C}=\mathrm{d} \times \mathrm{r}
\end{aligned}
$$

Em que:

$\mathrm{N}_{\mathrm{d}}=$ número de pares de dados;

$\mathrm{P}_{\mathrm{i}}=$ valor de $\mathrm{ET}_{0}$ estimado pelo método avaliado $(\mathrm{mm})$;

$\mathrm{O}_{\mathrm{i}}=$ valor de $\mathrm{ET}_{0}$ estimado pelo método padrão FAO Penman - Monteith (mm); $\mathrm{EQM}=$ erro quadrado médio $\left(\mathrm{mm}^{2}\right)$; $\overline{\mathrm{O}}=$ média dos valores $\mathrm{O}_{\mathrm{i}}(\mathrm{mm})$.

$\mathrm{O}$ índice $\mathrm{C}$ é obtido pelo produto do índice de precisão (coeficiente de correlação, r) pelo índice de concordância (d), sendo o critério de interpretação do desempenho dos métodos de estimativa da ETo pelo índice "C" foi proposto por CAMARGO e SENTELHAS (1997).
Além desses indicadores relatados acima, foram calculadas as médias, desvios padrão $(\sigma)$, coeficiente de variação $(\mathrm{cv})$, valores máximos e mínimos, tanto para o método padrão quanto para cada um dos outros modelos avaliados. Gráficos relativos à correlação também subsidiaram as comparações, objetivando auxiliar a análise visual.

As constantes empíricas dos modelos de Hargreaves, Makkink e Camargo, foram ajustadas em relação ao método padrão FAO, obtendo-se assim constantes exclusivas para Formiga - MG relativas a ambos os métodos. $\mathrm{O}$ ajuste foi realizado a partir da minimização dos erros, usando o pacote Solver do Microsoft Excel $^{\circledR}$ e o 
método de solução GRG não linear. As mesmas avaliações realizadas para estes três

\section{RESULTADOS E DISCUSSÃO}

A comparação dos métodos avaliados com o método padrão Penman-Monteith FAO, é apresentada na Tabela 1, em que pode ser observado que dentre os métodos não padrão, a maior média de $\mathrm{ET}_{0}$ diária foi obtida com o método de Hargreaves, enquanto que a menor pelo o método de Makkink ajustado, sendo 4,75 $\mathrm{mm}$ e 3,25 $\mathrm{mm}$, respectivamente. Porém, o método não padrão que apresentou média mais próxima ao método de Penman-Monteith FAO foi o de Camargo ajustado.

Dentre os valores encontrados para a estimativa do desvio padrão $(\sigma)$, o maior foi para método de Makkink e o que apresentou métodos não ajustados foram feitas após o ajuste. o menor desvio-padrão foi o de Camargo, sendo 1,26 e 0,29, respectivamente. Dentre os valores calculados para o coeficiente de variação (cv) o maior foi obtido para o modelo de Makkink $(0,33)$, enquanto que, os menores para os métodos de Camargo e o de Camargo ajustado, pois ambos obtiveram os mesmos valores $(0,07)$. Há de se salientar que um menor coeficiente de variação para os valores calculados de $\mathrm{ET}_{0}$ não inferem em dados com boa acurácia, já que é normal e esperado que a $\mathrm{ET}_{0}$ diária para um município com as características climáticas como o de Formiga, varie ao longo do ano.

Tabela 1. Índices estatísticos para as relações entre a ETo estimada pelo método de PenmanMonteith FAO e demais métodos avaliados

\begin{tabular}{cccccccc}
\hline & FAO & Hargreaves & $\begin{array}{c}\text { Hargreaves } \\
\text { ajustado }\end{array}$ & Makkink & $\begin{array}{c}\text { Makkink } \\
\text { ajustado }\end{array}$ & Camargo & $\begin{array}{c}\text { Camargo } \\
\text { ajustado }\end{array}$ \\
\hline Média & 3,38 & 4,75 & 3,37 & 3,77 & 3,25 & 3,97 & 3,40 \\
$\sigma$ & 0,79 & 0,89 & 0,63 & 1,26 & 1,05 & 0,29 & 0,30 \\
cv & 0,23 & 0,19 & 0,19 & 0,33 & 0,32 & 0,07 & 0,08 \\
Máximo & 5,50 & 6,46 & 4,59 & 6,11 & 5,20 & 4,65 & 3,99 \\
Mínimo & 1,17 & 1,68 & 1,19 & 0,29 & 0,34 & 2,60 & 2,22 \\
r & & 0,75 & 0,75 & 0,83 & 0,83 & 0,62 & 0,62 \\
p-valor & & 0,00 & 0,00 & 0,00 & 0,00 & 0,00 & 0,00 \\
EAM & & 1,37 & 0,40 & 0,68 & 0,50 & 0,74 & 0,53 \\
EQM & & 2,24 & 0,85 & 0,72 & 0,37 & 0,76 & 0,44 \\
REQM & 1,50 & 0,52 & 0,85 & 061 & 0,87 & 0,66 \\
d & 0,55 & 0,85 & 0,83 & 0,88 & 0,56 & 0,55 \\
c & & 0,42 & 0,64 & 0,69 & 0,73 & 0,35 & 0,34 \\
\hline
\end{tabular}

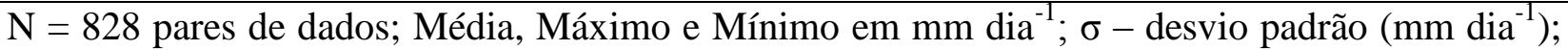
cv - coeficiente de variação; $r$ - coeficiente de correlação; EAM - erro absoluto médio (mm dia ${ }^{-}$ ${ }^{1}$ ); EQM- erro quadrado médio $\left(\mathrm{mm}^{2} \mathrm{dia}^{-2}\right)$, REQM - raiz quadrada do erro quadrado médio (mm $\left.\mathrm{dia}^{-1}\right) ; \mathrm{d}$ - índice de concordância; $\mathrm{c}$ - índice de confiança.

Para o coeficiente de correlação, $r$, foi obtido o maior valor para os modelos de Makkink e Makkink ajustado e o de menor valor nos métodos de Camargo e Camargo ajustado, sendo estes 0,83 e 0,62, respectivamente. Portanto analisando este indicador, os métodos de Makkink e Makkink ajustado são os que mais se aproximam da $\mathrm{ET}_{0}$ calculada pelo método FAO. Além disso, os p-valores obtidos para as correlações foram todos iguais a zero, 
portanto, sendo significativas as correlações positivas a um nível de significância de $1 \%$.

Dentre todos os métodos avaliados, o que apresentou maior valor para o erro absoluto médio, EAM, foi o modelo de Hargreaves $(1,37)$ enquanto que o de menor valor foi o método de Makkink ajustado $(0,50)$. Com isso, tem-se que o método de Hargreaves não representa bem a $\mathrm{ET}_{0}$ no município de Formiga, enquanto que o de Makkink após ajuste se aproxima mais das evapotranspirações de referência padrão.

Com relação aos modelos de Hargreaves, Makkink e Camargo antes e após o ajuste, verificou-se que se faz importante a análise dos parâmetros empíricos para cada localidade em que se deseja utilizá-los, a fim de se reduzir o EAM. Como observado na Tabela 1, para o modelo de Hargreaves, o EAM após o ajuste passou de 1,37 para 0,40. Já para o método de Makkink a redução foi de 0,68 para 0,50. E para o método de Camargo a redução foi de 0,74 para 0,53 .

Os coeficientes empíricos ajustados para os métodos de Hargreaves, Makkink e Camargo, para o município de Formiga MG, são mostrados na Tabela 2, sendo dois os coeficientes relativos ao método de Makkink.

Tabela 2. Coeficientes empíricos ajustados para os métodos de Hargreaves, Makkink e Camargo para estimativa da evapotranspiração de referência, Formiga, MG, Brasil.

\begin{tabular}{ccc}
\hline Método & Coeficiente original & Coeficiente proposto para Formiga - MG \\
\hline Hargreaves & 0,0023 & 0,001632 \\
Makkink & 0,61 & 0,509445 \\
& $-0,12$ & 0 \\
Camargo & 0,01 & 0,008566 \\
\hline
\end{tabular}

A comparação, por correlação linear, entre a evapotranspiração de referência diária obtida pelo método Penman-Monteith

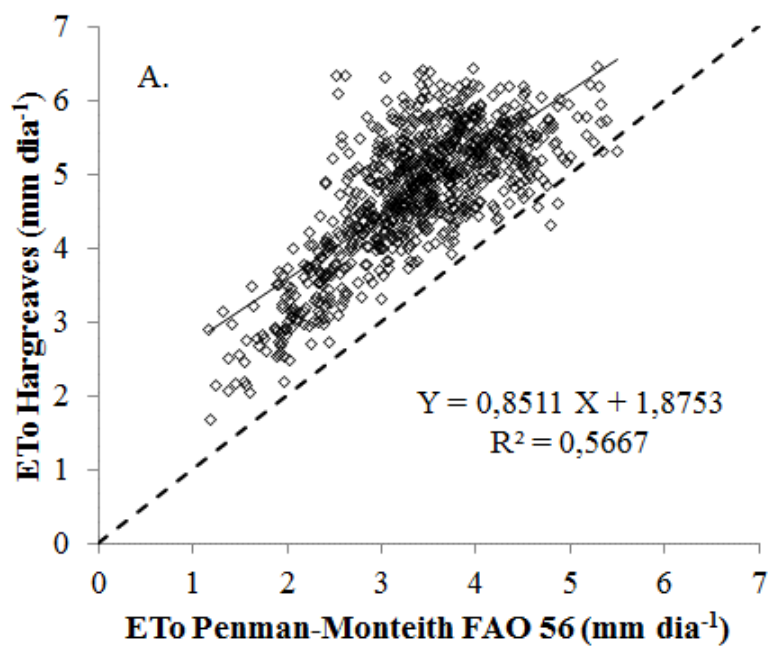

FAO e os métodos de Hargreaves e Hargreaves ajustado é apresentada na Figura 1 .

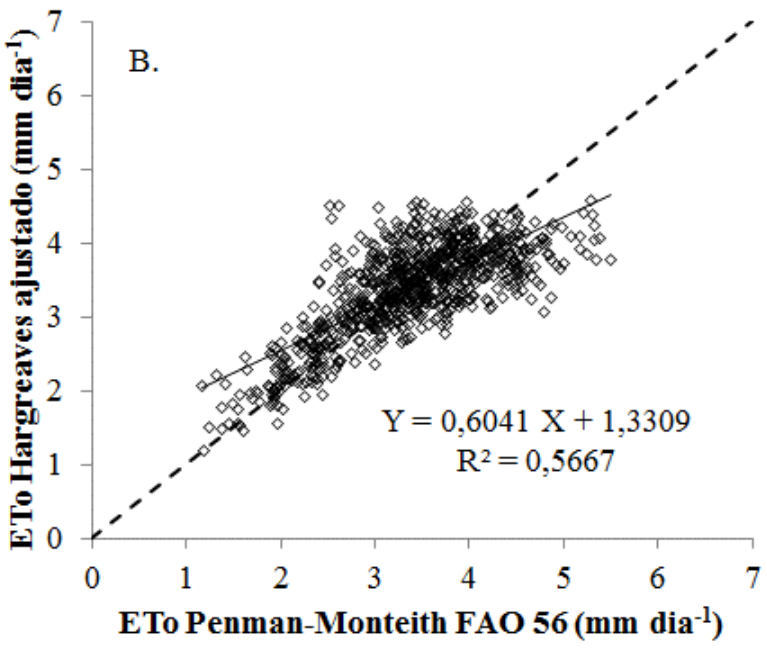

Figura 1. Evapotranspiração de referência diária estimada pelo método Penman-Monteith FAO comparada por correlação aos métodos de Hargreaves (A) e Hargreaves ajustado (B).

Observa-se na Figura 1A, que a linha de regressão linear entre o método de Penman-Monteith FAO e o método de
Hargreaves, se encontra totalmente acima da reta $1: 1$, demonstrando assim, que o método de Hargreaves superestima a 
evapotranspiração de referência diária em comparação com o método padrão. Percebese também que os pares de pontos se mostram dispersos, obtendo um coeficiente de determinação $\left(\mathrm{R}^{2}\right)$ igual a 0,56 .

$\mathrm{O}$ índice de concordância (d), que indica a concordância entre os dados observados e os estimados, como mostrado na Tabela 1, foi de 0,55. Enquanto que o índice de confiança (c) que evidencia o desempenho foi de 0,42 , classificando $o$ método como de "ruim", conforme critério de CAMARGO e SENTELHAS (1997). Este modelo se baseia apenas na temperatura do ar como variável climatológica e obtém melhores resultados para regiões de clima seco, não se ajustando assim muito bem para o município de Formiga.

BORGES JÚNIOR et al. (2012), ao estimar a evapotranspiração de referência por diversos métodos, em Garanhuns - PE, obtiveram que, dentre os métodos com base em temperatura por eles avaliados, aquele que gerou menor EAM foi o de Hargreaves, sendo 0,48 para primavera-verão e 0,36 para outono-inverno, resultados próximos aos obtidos neste trabalho. Porém, os autores encontraram valores de " $C$ " de 0,72 e 0,84 , respectivamente, enquanto para Formiga, o índice de confiança obtido foi menor.

O método de Hargreaves foi desenvolvido para clima semiárido, e como se baseia apenas em dados de temperatura, espera-se que este método superestime a $\mathrm{ET}_{0}$ em climas úmidos (SUBBURAYAN et al., 2011), como mostrado na Figura 1A. Deste modo, o ajuste deste método para as condições climáticas locais, se mostra importante. MAEDA et al. (2011) ao avaliarem a eficiência de métodos de estimação da $\mathrm{ET}_{0}$ encontraram que dentre os modelos estudados, o de Hargreaves foi $\mathrm{o}$ mais apropriado para as condições climáticas presentes na região Sul do Kenya, no continente Africano.

O método de Hargreaves ajustado (Figura 1B) obteve resultados melhores, em relação ao mesmo sem calibração, estando a linha de regressão mais próxima da reta de comparação 1:1. Mesmo com o coeficiente de determinação sendo mantido em 0,56 , os índices de concordância e o de confiança melhoraram relevantemente, sendo eles 0,85 e 0,64 , respectivamente.

O modelo continua a superestimar alguns valores de $\mathrm{ET}_{0}$ e a subestimar outros, em comparação com o método padrão. Porém seu desempenho passou de "ruim" para "mediano", segundo CAMARGO e SENTELHAS (1997). Isso mostra que o ajuste permitiu uma melhora na eficácia da estimativa da evapotranspiração de referência diária.

Não corroborando com o obtido neste estudo, BORGES JÚNIOR et al. (2012) não obtiveram melhora evidente do método de Hargreaves após calibração, aumentando de 0,72 a 0,77 para estação de primavera-verão e 0,84 a 0,86 para outono-inverno.

FRANÇA NETO et al. (2011), ao realizarem a comparação entre métodos simplificados de estimativa da $\mathrm{ET}_{0}$ em algumas regiões produtoras de café do Brasil, tiveram como resultado que $\mathrm{O}$ método de Hargreaves apresentou maior aproximação ao método Penman-Monteith FAO para estimativa da evapotranspiração de referência, quando comparado ao de Blaney-Criddle. Além disso, estes autores concluíram que o ajuste local dos métodos simplificados proporcionou melhoria significativa da estimativa de $\mathrm{ET}_{0}$, assim como o obtido neste trabalho.

Já a comparação entre a $\mathrm{ET}_{0}$ pelo método da FAO e os de Makkink e Makkink ajustado podem ser verificadas na Figura 2. 

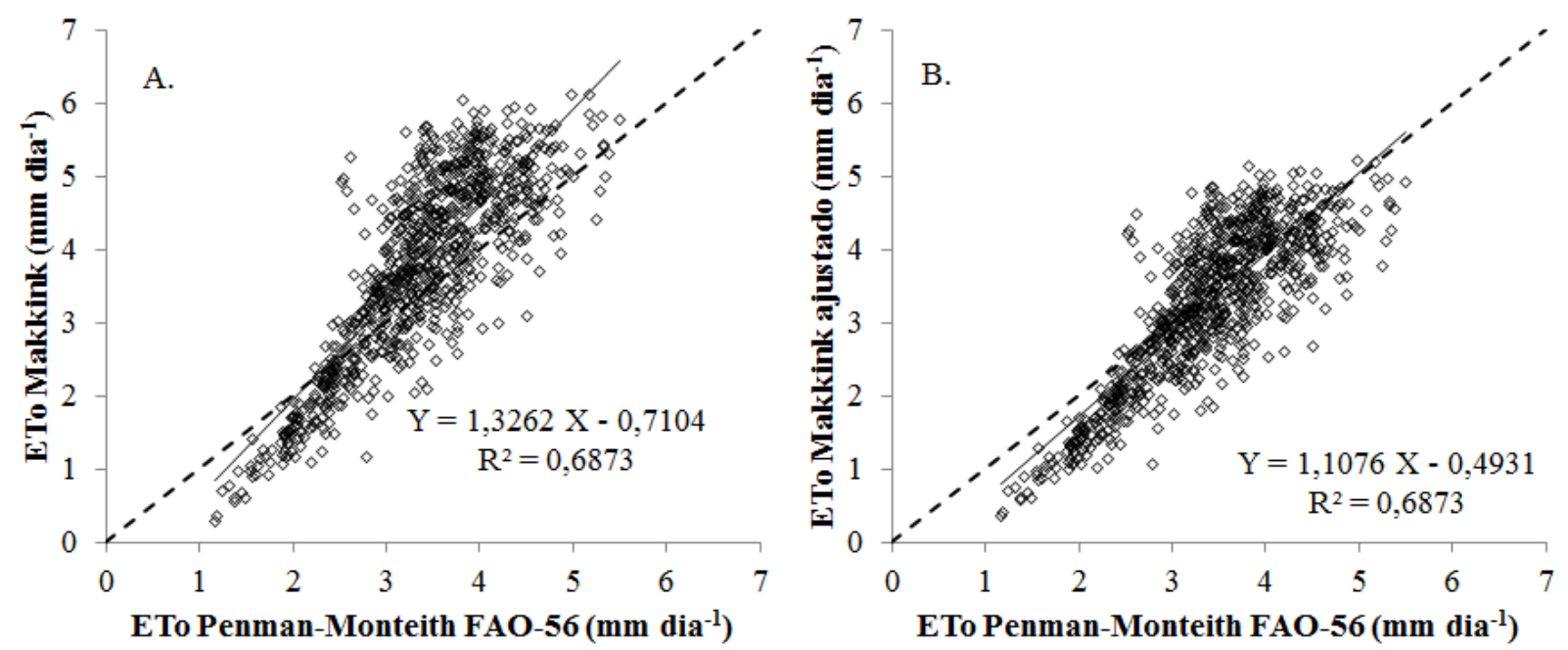

Figura 2. Evapotranspiração de referência diária estimada pelo método Penman-Monteith FAO comparada por correlação aos métodos de Makkink (A) e Makkink ajustado (B).

O modelo de Makkink apresentou menor dispersão dos pares de pontos, porém ele subestima a $\mathrm{ET}_{0}$ diária, quando esta, calculada pelo método padrão, é inferior a aproximadamente $2,5 \mathrm{~mm} \quad \mathrm{dia}^{-1}$, e superestima para valores acima deste. Este método exibiu um índice de concordância melhor que o de Hargreaves, com valor de 0,83 (Tabela 1) e um índice de confiança igual a 0,69 , em relação ao método padrão FAO, mostrando assim, um desempenho classificado como "bom".

MELO e FERNANDES (2012), ao avaliarem métodos empíricos para estimativa da $\mathrm{ET}_{0}$ no município de Uberaba, MG, concluíram que em função dos resultados obtidos dos índices de performance, seria recomendado o uso do modelo de Makkink para estimar a $\mathrm{ET}_{0}$ naquele município, corroborando com o observado neste estudo. Além disso, os resultados apresentados por FERNANDES et al. (2012) mostraram que a evapotranspiração de referência determinada pelos métodos de Hargreaves, PriestleyTaylor and Makkink apresentaram maiores concordâncias com o obtido com o uso da equação Penman-Monteith FAO, em Campos dos Goytacazes/RJ.

Em contraposição ao ocorrido neste trabalho, LACERDA e TURCO (2015), ao estudarem a determinação da $\mathrm{ET}_{0}$ do município de Uberlândia utilizando diferentes métodos, obtiveram que o modelo de Makkink subestimou a $\mathrm{ET}_{0}$ em $38 \%$ quando os valores foram comparados aos obtidos utilizando o método de PenmanMonteith.

Além deste trabalho, PILAU et al. (2012) observaram que o método de Makkink subestimou a $\mathrm{ET}_{0}$ em relação ao método de Penman-Monteith nos municípios de Frederico Westphalen e Palmeira das Missões, RS, na maioria dos meses do ano. Enquanto que, CAVALCANTE JÚNIOR et al. (2011), em condições semiáridas do Nordeste brasileiro, também determinaram que o método de Makkink subestimou a $\mathrm{ET}_{0}$ em relação ao método de Penman-Monteith tanto em meses úmidos quanto secos, sendo mais pronunciado este padrão na estação seca.

O método de Makkink ajustado (Figura 2B) obteve o melhor resultado de todos, apresentando menor dispersão dos pares de pontos, estando estes mais próximos da linha de regressão e da reta de comparação 1:1. O desempenho dele em relação ao método padrão foi classificado como "bom" (CAMARGO e SENTELHAS, 1997), com valor de 0,73 do índice de confiança, apresentando índice de 
concordância e coeficiente de determinação de 0,88 e 0,68 , respectivamente.

Realizar a calibração para o modelo de Makkink se mostrou eficaz já que, apesar do $\mathrm{R}^{2}$ ter permanecido o mesmo, os outros indicadores, c e d, melhoraram. Assim, após a adequação, se obteve a melhor combinação de valores dentre todos os métodos analisados.

O método de Penman-Monteith FAO é o mais preciso por considerar todas as variáveis físicas e climatológicas que influenciam $\quad \mathrm{o}$ fenômeno da evapotranspiração. Porém, o método de
Makkink ajustado mostrou-se satisfatório para a estimativa da $\mathrm{ET}_{0}$ diária para o município de Formiga, MG. É importante ressaltar que esse modelo leva em consideração a radiação solar global além da temperatura do ar, portanto, demonstra que certas variáveis consideradas na equação influenciam no desempenho dos métodos, dentre outros fatores.

Já a evapotranspiração de referência diária pelo método Penman-Monteith FAO comparado por correlação linear aos métodos de Camargo e Camargo ajustado, é apresentada na Figura 3.
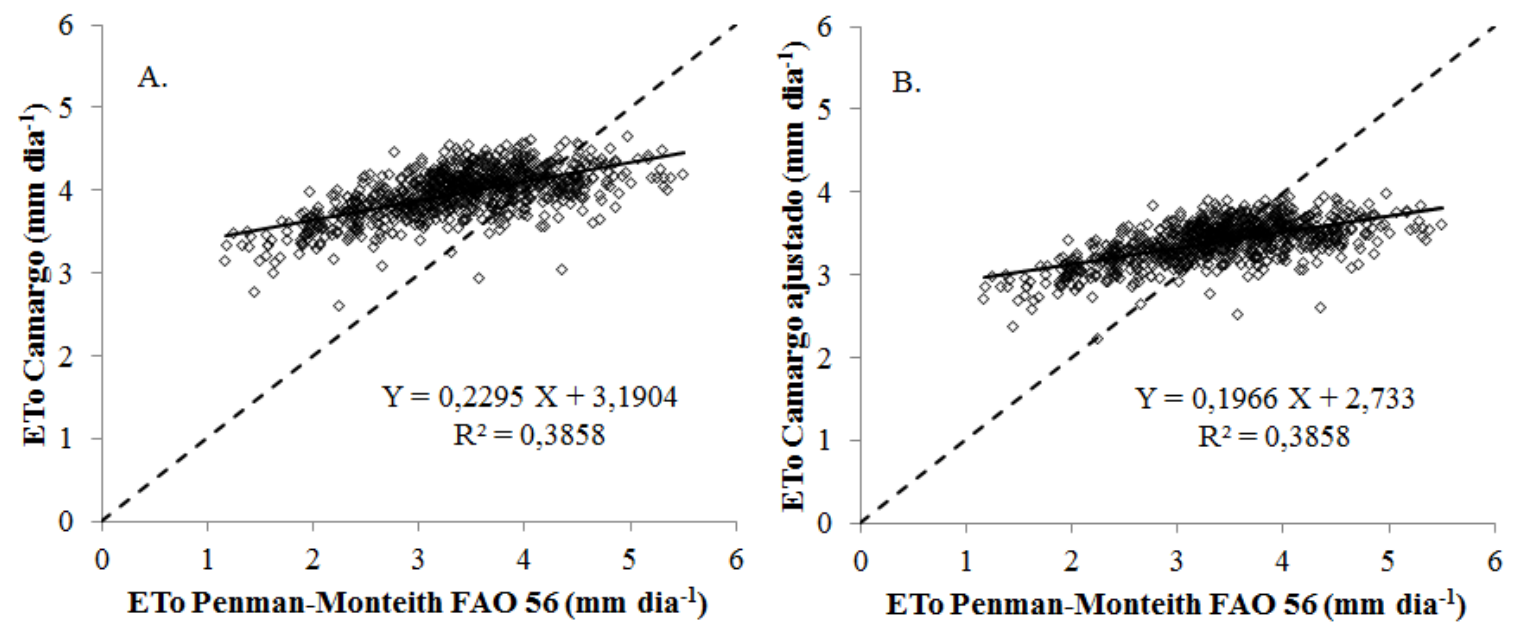

Figura 3. Evapotranspiração de referência diária estimada pelo método Penman-Monteith FAO comparada por correlação aos métodos de Camargo (A) e Camargo ajustado (B).

Verifica-se na Figura 3 que o método de Camargo superestima a $\mathrm{ET}_{0}$ até aproximadamente $4 \mathrm{~mm} \mathrm{dia}^{-1}$ e depois os subestima, porém, manifestando uma tendência de valores de evapotranspiração de referência entre 3 e $4 \quad \mathrm{~mm} \quad \mathrm{dia}^{-1}$, independentemente do valor obtido para o método padrão. Neste método, obteve-se em valor de $\mathrm{c}$ igual a 0,35 , desempenho classificado como "péssimo" (CAMARGO e SENTELHAS, 1997).

Resultados obtidos por MELO e FERNANDES (2012) em seu trabalho sobre a estimativa da $\mathrm{ET}_{0} \mathrm{em}$ Uberaba, $\mathrm{MG}$, não corroboram com os avaliados neste estudo, já que os autores anteriormente citados, encontraram valor para o índice " $\mathrm{C}$ " para o método de Camargo classificado como "bom".

Os mesmos autores ainda concluíram em seu estudo que os métodos de Makkink, Camargo e Hargreaves, usando apenas elementos meteorológicos como temperatura e radiação solar como variáveis de entrada mostraram melhores performances comparados a outros métodos usando um número maior de varáveis, como JensenHaise, Linacre e Radiação Solar.

Após a calibração, observa-se que houve uma pequena melhora na disposição 
dos pares de pontos em relação à reta $1: 1$, porém ele continua a superestimar a $\mathrm{ET}_{0}$ para valores aproximadamente inferiores a 3,5 $\mathrm{mm} \mathrm{dia}^{-1}$ e a subestimá-la após. Seu índice de confiança foi de 0,34 , continuando a ser classificado como "péssimo" por CAMARGO e SENTELHAS (1997).

O método de Camargo é baseado na temperatura média do ar, e revelou-se ineficiente para estimar a evapotranspiração de referência diária do município de Formiga, pois apresentou a pior combinação de valores de testes de avaliação dentre

\section{CONCLUSÃO}

Dentre todos os métodos avaliados para a estimativa diária da evapotranspiração de referência no município de Formiga MG, o modelo ajustado de Makkink foi o que apresentou melhor desempenho. Este é o que mais se aproxima do método de Penman-Monteith FAO, considerando os valores do erro absoluto médio, do índice de

\section{REFERÊNCIAS}

ALENCAR, L. P.; SEDIYAMA, G. C.; WANDERLEY, H. S.; ALMEIDA, T. S.; DELGADO, R. C. Avaliação de métodos de estimativa da evapotranspiração de referência para três localidades no norte de Minas Gerais. Engenharia na Agricultura, Viçosa, v.19 n.5, p.437-449, 2011.

ALLEN, R. G.; PEREIRA, L. S.; RAES, D.; SMITH, M. Crop evapotranspiration: guidelines for computing crop water requirements. Rome: FAO, 1998. 297p. (FAO Irrigation and Drainage Paper 56).

ALVARES, C. A.; STAPE, J. L.; SENTELHAS, P. C.; GONÇALVES, J. L. M.; SPAROVEK, G. Köppen's climate classification map for Brazil. Meteorologische Zeitschrift, Stuttgart, v.22, n.6, p.711-728, 2013. todos os outros métodos avaliados. Há de se salientar, que o método de Camargo foi inicialmente proposto para determinação da Evapotranspiração de Referência para períodos a partir de sete dias, fato este que contribuiu para sua baixa acurácia.

Adicionalmente, FERNANDES et al. (2012) citam que, mesmo o método de Camargo utilizando a radiação solar no topo da atmosfera, esta informação não leva em conta a redução da energia até atingir a superfície, graças à interação entre radiação e atmosfera.

confiança, do índice de concordância e do coeficiente de determinação, enquanto que, o método de Camargo exibiu as piores avaliações dos testes de desempenho em relação ao método padrão, o que o torna insatisfatório para a estimação da $\mathrm{ET}_{0}$ diária do município.

BORGES JÚNIOR, J. C. F. Métodos de estimativa da evapotranspiração de referência diária para a microrregião de Garanhuns, PE. Revista Brasileira de Engenharia Agrícola e Ambiental, Campina Grande, v.16, n.4, p.380-390, 2012.

CAMARGO, A.P.; SENTELHAS, P.C. Avaliação do desempenho de diferentes métodos de estimativa da evapotranspiração potencial no Estado de São Paulo, Brasil. Revista Brasileira de Agrometeorologia, Santa Maria, v.5, n.1, p.89-97, 1997.

CAVALCANTE JÚNIOR, E. G.; OLIVEIRA, A. D.; ALMEIDA, B. M.; ESPÍNOLA SOBRINHO, J. Métodos de estimativa da evapotranspiração de referência para condições do Semiárido 
Nordestino. Semina: Ciências Agrícolas, Londrina, v.32, supl. 1, p.1699-1708, 2011.

FERNANDES, L. C.; PAIVA, C. M.; ROTUNNO FILHO, O. C. Evaluation of six empirical evapotranspiration equations case study: Campos dos Goytacazes/RJ. Revista Brasileira de Meteorologia, São Paulo, v.27, n.3, p.272-280, 2012.

FRANÇA NETO, A. C.; MANTOVANI, E. C.; VICENTE, M. R.; VIEIRA, G. H. S.; SEDIYAMA, G. C.; LEAL, B. G. Comparação entre métodos simplificados de estimativa da evapotranspiração de referência (ET0) para regiões produtoras de café brasileiras. Coffee Science, Lavras, v.6, n.2, p.159- 171, 2011.

LACERDA, Z. C.; TURCO, J. E. P. Estimation methods of reference evapotranspiration (ETo) for Uberlândia MG. Engenharia Agrícola, Jaboticabal, v.35, n.1, p.27-38, 2015.

MAEDA, E. E.; WIBERG, D. A.; PELLIKKA, P. K. E. Estimating reference evapotranspiration using remote sensing and empirical models in a region with limited ground data availability in Kenya. Applied Geography, Amsterdam, v.31, n.1, p.251258, 2011.

MELO, G. L.; FERNANDES, A. L. T. Evaluation of empirical methods to estimate reference evapotranspiration in Uberaba, state of Minas Gerais, Brazil. Engenharia Agrícola, Jaboticabal, v.32, n.5, p.875-888, 2012.

PEREIRA, D. R.; YANAGI, S. N. M.; MELLO, C. R.; SILVA, A. M.; SILVA, L. A. Desempenho de métodos de estimativa da evapotranspiração de referência para a região da Serra da Mantiqueira, MG. Ciência Rural, Santa Maria, v.39, n.9, p.2488-2493, 2009.
PILAU, F. $\quad$ G.; $\quad$ BATTISTI, R.; SOMAVILLA, L.; RIGHI, E. Z. Desempenho de métodos de estimativa da evapotranspiração de referência nas localidades de Frederico Westphalen e Palmeira das Missões - RS. Ciência Rural, Santa Maria, v.42, n.2, p.283-290, 2012.

SUBBURAYAN, S., MURUGAPPAN, A., MOHAN, S. Modified Hargreaves Equation for Estimation of ETo in a Hot and Humid Location in Tamilnadu State, India. International Journal of Engineering Science and Technology, Tehran, v.3, n.1, 2011.

WILLMOTT, C. J. Some comments on the evaluation of model performance. Bulletin American Meteorological Society, v.30, p.1309-1310, 1982. 